\title{
The first detection of Salmonella enterica subspecies enterica serovar Choleraesuis in farm-raised pigs in Serbia, 2019
}

\author{
Bozidar Savic ${ }^{1}$, Nemanja Zdravkovic ${ }^{1}$, Oliver Radanovic ${ }^{1}$, Nemanja Jezdimirović ${ }^{2}$, \\ Branislav Kureljusic ${ }^{1}$, and Ognjen Stevancevic ${ }^{3}$ \\ ${ }^{1}$ Institute of Veterinary Medicine Belgrade, Serbia \\ ${ }^{2}$ Institute of Veterinary Medicine of Serbia \\ ${ }^{3}$ Agriculture Faculty Department for Veterinary Medicine University of Novi Sad
}

October 1, 2020

\begin{abstract}
Salmonella enterica subspecies enterica serovar Choleraesuis is rarely detected in Europe, but the clinical disease in pigs, was reported in wild boars. Salmonellosis caused by S. Choleraesuis has never been confirmed in Serbia, as in many other countries in Europe. In April 2019, on one large farrow-to-finish pig farm, an increase in mortality in weaned piglets with lethargy, anorexia, pyrexia and respiratory distress was reported. Gross pathology revealed dermal cyanosis, mesenteric lymphadenopathy, splenomegaly, hepatomegaly, interstitial pneumonia and colitis. By direct culturing of lung, liver, spleen and lymph nodes S. Choleraesuis variant Kunzendorf was isolated. This is the first report on the detection of S. Choleraesuis in domestic pigs in Serbia and re-appearance of salmonellosis due to S. Choleraesuis after decades of absence of disease on pig farms in Europe, which provides useful information about the epidemiology of this agent on the European continent. However, the definitive source for this outbreak and origin of infection still have to be elucidated.
\end{abstract}

The first detection of Salmonella enterica subspecies enterica serovar Choleraesuis in farm-raised pigs in Serbia, 2019

Bozidar Savic $^{1,2^{*}}$, Nemanja Zdravkovic ${ }^{1}$, Oliver Radanovic $^{1}$, Nemanja Jezdimirovic ${ }^{1}$, Branislav Kureljusic ${ }^{1}$, Ognjen Stevancevic ${ }^{2}$

${ }^{1}$ Institute of Veterinary Medicine Belgrade, J. Janulisa 14, 11000 Belgrade, Serbia

${ }^{2}$ Agriculture Faculty Department for Veterinary Medicine University of Novi Sad, Dositej Obradovic Square 8, 21000 Novi Sad, Serbia

*Corresponding author. Bozidar Savic, Institute of Veterinary Medicine Belgrade, J. Janulisa 14, 11000 Belgrade, Serbia. Tel.: + 381112851 065, Fax: + 381112851 065, E-mail address: savic.bozidar@hotmail.com (Savic. B)

\section{Summary}

Salmonella enterica subspecies enterica serovar Choleraesuis is rarely detected in Europe, but the clinical disease in pigs, was reported in wild boars. Salmonellosis caused by S. Choleraesuis has never been confirmed in Serbia, as in many other countries in Europe. In April 2019, on one large farrow-to-finish pig farm, an increase in mortality in weaned piglets with lethargy, anorexia, pyrexia and respiratory distress was reported. Gross pathology revealed dermal cyanosis, mesenteric lymphadenopathy, splenomegaly, hepatomegaly, interstitial pneumonia and colitis. By direct culturing of lung, liver, spleen and lymph nodes S. Choleraesuis variant Kunzendorf was isolated. This is the first report on the detection of S. Choleraesuis in domestic pigs 
in Serbia and re-appearance of salmonellosis due to S. Choleraesuis after decades of absence of disease on pig farms in Europe, which provides useful information about the epidemiology of this agent on the European continent. However, the definitive source for this outbreak and origin of infection still have to be elucidated.

Keywords

Salmonella Choleraesuis; outbreak investigation; farm pigs; Serbia

Introduction

Host-adapted S. Choleraesuis is isolated almost exclusively from diseased swine and is usually manifested as septicemia (Griffith et al., 2019). S. Choleraesuis var. Kunzendorf is currently highly prevalent in North America and Asia (Luk-in et al., 2018), but is rare in Australia and the European Union (EU) (Fedorka-Cray et al., 2000). In Europe, S. Choleraesuis is not considered a dominant serovar, in slaughter pigs and breeding herds and only a few outbreaks have been reported, among pig herds within the last decades; 1999-2000 and 2012-2013 (Baggesen et al., 2000; Pedersen et al., 2015) (in Denmark), but it is occasionally reported, such as in 2009 (EFSA 2008, 2009), 2011 (EFSA 2013) and 2014 (EFSA 2014) with a low frequency in a few European countries. However, several cases of outbreak of the septicaemic form, caused by S. Choleraesuis var. Kunzendorf, were reported in Europe in wild boars: in Spain in 1999 (Perez et al., 1999); in Thuringia between 2006 and 2008 (Methner et al., 2010); in central-western Spain between 2010 and 2016 (Gil Molino et al., 2019), in Italy from February 2012 until June 2015 (Longo et al., 2019); and in the German federal state North Rhine-Westphalia in 2017 (Methner et al., 2018). In Serbia, the most common Salmonella serovars in pigs are Salmonella enterica serovar Typhimurium, S. Derby, S. Infantis and S. Enteritidis (Stojanac et al., 2014; Kureljusic et al., 2017). These serovars may also cause clinical salmonellosis in pigs, however except in sporadic reports, the extent of clinical salmonellosis in pigs in Serbia is uncertain. Furthermore, in Serbia, until 2019 there was no reported salmonellosis caused by S. Choleraesuis. Here, we describe clinical symptoms, pathological changes and microbiological features of swine Salmonellosis caused by Salmonella Choleraesuis in weaned pigs on a farrow-to-finish pig farm in Serbia, as well as determining the in vitro susceptibilities of the S. Choleraesuis isolate against commonly used antimicrobials.

Material and methods

In April 2019, on a farrow-to-finish pig farm in south-west Vojvodina province in the northern region of Serbia, an increase in mortality from 2.3 to $5 \%$ in weaned piglets was reported. The herd had an inventory of 1300 sows on a single-site, and the farm practices continuous flow operations on the basis of weekly management. In every segment of the production cycle the "all in-all out" principle is used, and all facilities are cleaned, washed and disinfected after emptying, before being re-populated in 3-4 days. There were three nursery buildings on the farm housing approximately 2000 weaned piglets per building, as well as additional facilities for housing of other phases of swine production. The piglets were fed with a complete feed mixture for weaned pigs purchased from local feed mills, and the farm also prepared and used its own feed. The farm vaccinates against neonatal colibacillosis and Clostridium infections, Mycoplasma hyopneumoniae (Mh), Classical Swine Fever (CSF), Aujeszky's disease, erysipelas, porcine parvovirus (PPV) Porcine circovirus 2 (PCV2) and Porcine Reproductive and Respiratory Syndrome Virus (PRRSV) (sows at weaning). In the previous 8 months the farm had experienced an episode of a severe outbreak of Porcine Epidemic Diarrhea Virus (PEDV) infection. Also, in the previous 1.5 years, problems related to mycotoxicoses in different phases, had regularly occurred on the farm. The history of salmonellosis on the farm was unknown. During the previous 2 years, the farm had imported about 200 gilts and 7 boars from the EU, but the farm also regularly traded pigs with other swine farms within Serbia.

The outbreak of disease was observed in a nursery building in which there were 900 piglets size $15-20 \mathrm{~kg}$. About 120 piglets out of 900, displayed clinical symptoms including lethargy, inappetence, reluctance to move, and cyanosis of ears, extremities and abdomen, while approximately 50 piglets with such clinical symptomatology were also febrile with temperatures of $39.5-41.5^{\circ} \mathrm{C}$ and had shallow cough and hard breathing (thumping) giving the impression of severe pneumonia. The same clinical symptomatology was also observed in the other two nursery buildings a day later, and approximately 460 of, in total, 6000 weaned 
piglets at the farm showed a similar clinical picture. To control the outbreak, some management procedures (isolation of sick piglets), in the pens with clinical disease was implemented to reduce infection and contamination within nurseries, and antibiotic treatment was initiated. The disease outbreak in nurseries associated with observed clinical signs lasted for 3 weeks. Morbidity reached $10 \%$ among weaned piglets, and the mortality increased to approximately 5\%. Of the 460 diseased piglets, 152 died, reaching $100 \%$ morbidity, with a case fatality rate of $33 \%$.

In the examined piglets, a necropsy performed by veterinarians and a pathologist from the Veterinary Institute, at the farm, revealed emaciated carcasses and rough hair. Most carcasses showed distal cyanosis of skin, especially ears, nose, ventral neck, feet and ventral part of the abdomen. In piglets that had survived for a few days, the tips of ears were dry and dark-red, with sloughed portions (Figure 1a). Lymph nodes especially the mesenteric, were enlarged, dark purple and moist (Figure 1b). In most cases, the spleen was enlarged (splenomegaly), dark-purple and pulpy while the liver was moderately enlarged (hepatomegaly), frequently with scattered small ([?]1.5 mm) white spots (foci of parenchymal necrosis) (Figure 1b). In all cases, the lungs were resilient, non-collapsed slightly firm with red fluid that separated lobules, and lesions affected either the cranial or the apical lobes. Colitis was found in some piglets. The mucosae of the colon from those animals were slightly thickened and multifocally covered by adherent gray-yellow fibrinous membranes (Figure 1c). Samples of different organs (lungs, liver, spleen, and lymph nodes) from six animals were sent to the National Reference Laboratory for Salmonella (Institute of Veterinary Medicine, Belgrade) for Salmonella identification and serotyping. In addition, at the pathology department, tissue samples of these organs were fixed in 10\% neutral buffered formalin for histopathological examination. Tissue sections were embedded in paraffin, cut at $5 \mu \mathrm{m}$, stained with $\mathrm{H} \& \mathrm{E}$ and examined under the microscope.

All samples were cultured on blood agar, McConkey agar, and xyloselysine-desoxicholate agar (XLD) in aerobic conditions for $24 \mathrm{hr}$. at $37^{\circ} \mathrm{C}$. Compatible colonies were confirmed by detection of invE and invA genes by PCR (Stone et al., 1994) and biochemically using BBL Crystal Identification Systems Enteric/Nonfermenter id kit. Serogroup identification was performed by using Salmonella Sero-Quick Group kit (SSI Diagnostica). The identification of serotypes was conducted according to the Kauffmann-White scheme (Popoff 2001). Salmonella isolates that belonged to $\mathrm{C} 1$ group, serotype 6,7:c:1,5 were further sequenced for the fliC gene using flinC-F and flinC-R primers described by Chiu et al., 2005. The sequences were queried in the NCBI Genbank base to identify best match with the fliC gene from Salmonella. The 871 base-pair sequence of the fliC flagellin gene of Salmonella from this study was submitted to the GenBank database under-accession number MN563751. For antimicrobial sensitivity and resistance testing, isolates were tested by disc diffusion against a panel of 12 antimicrobials from different families which are routinely used in farms (Table1).

Results and discussion

A tentative diagnosis of salmonellosis caused by S. Choleraesuis was based on clinical signs and postmortem lesions. The observed clinical symptoms indicated respiratory infections, and also fitted the clinical presentation of salmonellosis due to S. Choleraesuis. Lesions detected by necropsy of piglets that died, indicated a septicaemic processes, and this was confirmed by the isolation of S. Choleraesuis from different organs in the examined animals. Congestion and foci of necrosis with interstitial infiltration with mononuclear cells in the liver (Figure 1d); diffuse interstitial pneumonia with septal thickening accompanied with suppurative bronchopneumonia, oedema and congestion in the lung; congestion, increase of white pulp and foci of necrosis in the spleen; hyperemia, with areas of cellular necrosis in the lymph nodes were the hallmarks of histology findings.

Six bacterial strains that were recovered from different animals directly without pre-enrichment in buffered peptone water were classified as S. Choleraesuis var. Kunzendorf by phenotypic serotyping and biochemical tests. All isolates were assigned to the antigenic formula 6,7:c:1,5 and were Vi capsular polysaccharide (Vi antigen) negative, showing the following biochemical features: dulcitol (-), H2S (+) and mucate (-). All isolates generated the $963 \mathrm{bp}$. PCR product of the fliC gene. The reverse primer FlinC-R amplified a unique sequence specific for S. Choleraesuis and S. Paratyphi C. Because S. Paratyphi C infects only humans and not swine, and as isolates were $\mathrm{Vi}$ antigen negative, the positive signal of the fliC gene from these isolates 
is conclusive for S. Choleraesuis. Regarding antibiotic groups, isolates showed resistance to penicillins, tetracyclines, phenicols, and streptomycin and could be considered multiresistant, while all isolates showed sensitivity to aminoglycosides, cephalosporins, quinolones, sulfonamides and colistin.

Our findings presented here show the first case of salmonellosis caused by S. Choleraesuis in farm-raised pigs in Serbia. In our case, the disease occurred in nurseries and that age of piglets has been described in the literature as the most susceptible to disease. Many studies (Chiu et al., 2004; Pedersen et al., 2015; Gil Molino et al., 2019) emphasize the importance of stress factors, such as weaning and grouping animals, the triggering effect of immunosuppressive viruses (such as PCV2, PRRSV) and the fact that the piglets aged 2-3 months have the lowest level of antibodies since birth (Tizard, 2009), as activators of various types of infections in weaned piglets. This also applies to Salmonella species, and the ability of the strains of Salmonella to persist for long periods of time in asymptomatic carriers, and then to be activated by stress factors. In this case, the farm had no known previous confirmed history of salmonellosis and it was not known whether S. Choleraesuis already persisted or not, in weaned piglets and on the farm at all. Furthermore, the farm applies vaccination against major and specific pig pathogens including vaccination of piglets against M. hyopneumoniae, PCV2, porcine pseudorabies virus (PRV), CSF-virus and erysipelas, and only a couple of months before the outbreak of the S. Choleraesuis there was an outbreak of severe PEDV infection. However, as we did not know the history of salmonellosis on the farm or whether S. Choleraesuis persists at all, it can only be speculated whether PEDV infection in this case has contributed to the severity of the S. Choleraesuis outbreak in weaned piglets. On the other hand, if asymptomatic S. Choleraesuis infection was already present (in small numbers) in weaned piglets, then its activation, and subsequent acute outbreak of the disease could be associated to the triggering effect of predisposing factors such as underlying diseases and conditions (e.g. mycotoxicoses), decreased antibody levels, especially the level of antibodies against PRRSV, (as only sows are vaccinated), and therefore the onset of active PRRSV infection in piglets (Wills et al., 2000), and stress-induced immuno-suppression. Also, an additional explanation for the outbreak of S. Choleraesuis in weaned piglets may be that S. Choleraesuis does not need any previous immunosuppressant infections to develop a pathological process, as recently described in outbreaks of S. Choleraesuis in wild boars in Spain and Italy (Gil Molino et al., 2018; Longo et al., 2019).

The outbreak of the disease from our study, was characterized by a high mortality rate of $33 \%$, which is the same rate reported in outbreaks of salmonellosis due to S. Choleraesuis in four Danish pig farms in 2012-2013, with the exception that in our case there were no obvious concomitant infections during the disease. Clinical symptoms and pathological findings of the affected piglets were fully compatible with those described in the literature for farm-raised weaned pigs, and comparable to those described in similar processes affecting wild boars (Methner et al., 2018; Gil Molino 2018), with addition of findings of colitis in several cases which is uncommonly a pathological feature of septicaemic salmonellosis caused by S. Choleraesuis.

The most likely source of infection in this case is live carrier pigs, although other sources such as feed, wild boars or even humans as passive vectors although unlikely, cannot be entirely ruled out (Hilbert et al., 2012). The farm had successively imported a large number of pigs (gilts and boars) in the previous two years from the EU, but it had also purchased pigs from farms in Serbia. Therefore, it is possible that during an import or by purchasing of pigs from farms in Serbia, S. Choleraesuis was introduced onto the farm, by undetected carrier animals. However, the lack of reports on S. Choleraesuis infections on pig farms from neighboring and many European countries does not allow us to hypothesize on the possible wider origin of the infection. Also, from the authors' knowledge, until now S. Choleraesuis has not been detected in Serbia, though owing to intensive imports of pigs into the country for the last two decades from many EU countries, we cannot with certainty exclude the possibility that S. Choleraesuis, undetected, could have been present in asymptomatic pigs on certain farms in the country, or in the wild boar population and even in the pig food chain, spread by carriers among pig herds all over the country, including spreading to the farm from this case, primarily due to insufficient detection of Salmonella on pig farms at the national level. On the other hand, clinical salmonellosis caused by S. Choleraesuis can hardly pass undetected on farms by either the owner or a veterinarian without reporting this and, thereby, allowing a persistent infection. It is generally accepted that S. Choleraesuis is rarely found in pig feed, and in Serbia there is no stock of wild boars in 
the area, and the farm from this case is relatively well managed, with a good biosecurity, and finally during epidemiological investigation we could find no evidence that the employees at the farm were the vectors of infection, so all these potential reservoirs of Salmonella were ruled out, as possible sources or vectors of the infection on the farm.

In conclusion, this is the first report on the septicaemic form of salmonellosis caused by S. Choleraesuis var. Kunzendorf, in farm-raised weaned pigs from Serbia, and beside a few outbreaks of S. Choleraesuis reported in Danish pig farms during 1999-2000 and 2012-2013, this is also the first report of S. Choleraesuis infection on pig farms in Europe in the last several decades. The infection on the farm was most likely introduced by live asymptomatic carrier pigs, either imported or originating from swine herds from Serbia. However, only molecular typing and comparison of our isolates with isolates from other European countries may allow a better conclusion on the potential source and tracing the origin of the infection which could not be done in the current investigation. Undetected, asymptomatic infection on the farm had probably persisted for some time and then was activated by stress-induced immuno-suppression, thus triggering the clinical outbreak of disease among weaned piglets. In spite of low-prevalence of S. Choleraesuis as a cause of salmonellosis in pig farms in Europe, it appears from this study that S. Choleraesuis could be underestimated as a pathogen in farm pigs, and that its prevalence, as an asymptomatic infection, may be higher than assumed owing to the fact that the number of wild boars, that serve as a most likely reservoir of S. Choleraesuis, has significantly increased on the European continent in the last decades, and that contacts of humans with the wild environment are more frequent, thus increasing the risk of occurrence and transmission of diseases to farmed pigs (Ruiz-Fons, 2017). Our finding of salmonellosis caused by S. Choleraesuis in farm-raised pigs reemphasizes the importance of hygiene and biosecurity measures in the herds for preventing the introduction of specific pathogens and spreading of diseases. Particular care must be taken towards trucks which deliver live pigs directly to the farm from an area, or pass through areas, of Europe or Serbia, where S. Choleraesuis may be present, which are not being properly cleaned and disinfected.

Acknowledgements

This work was partially funded by Serbian Ministry of Education, Science and Technological Development (contract number: 451-03-68/2020-14/200030). We thank the veterinary practitioners for the farm information and aid during necropsy and sampling as well as to the technical staff from the Department of Bacteriology and Pathology of the Institute of Veterinary Medicine Belgrade, Serbia.

Conflict of interest statement

All authors have declared no conflict of interest.

Ethical approval

The authors confirm that the ethical policies of the journal, as noted on the journals author guidelines page, have been adhered to. No ethical approval was required as this article describes field data and no live animals were handled to obtain tissues.

Data viability statement

The data that support the findings of this study are available from the corresponding author upon reasonable request. The sequence used to support the finding of this study is available in GenBank at https://www.ncbi.nlm.nih.gov reference number MN563751.

Orcid

Savic Bozidar: https://orcid.org/0000-0001-7705-6457

\section{References}

Baggesen, D. L., Christensen, J., Jensen, T. K., Skov, M. N., Sorensen, G., \& Sorensen, V. (2000). Udbrud af Salmonella enterica subsp. enterica serovar Choleraesuis var. Kunzendorf (S. Choleraesuia) i en dansk svinebesaetning. Dansk Veterinaertidsskrift, 83, 6-12. 
Chiu, C.H., Su, L.H., \& Chu, C. (2004). Salmonella enterica serotype Choleraesuis: Epidemiology, Pathogenesis, Clinical Disease, and Treatment. Clinical Microbiology Review, Vol 17, No. 2, 311-322. https://doi.org/10.1128/CMR.17.2.311-322.2004.

Chiu, T. H., Pang, J. C., Hwang, W. Z., \& Tsen, H. Y. (2005). Development of PCR Primers for the Detection of Salmonella enterica Serovar Choleraesuis Based on the fliC Gene. Journal of Food Protection, Vol. 68, No. 8, 1575-1580. https://doi.org/10.4315/0362-028X-68.8.1575.

EFSA (2008). Report of the Task Force on Zoonoses Data Collection on the analysis of the baseline survey on the prevalence of Salmonella in slaughter pigs, in the EU, 2006-20071. Part A: Salmonella prevalence estimates. EFSA Journal, 135, 1-111. https://doi.org/10.2903/j.efsa.2008.135r.

EFSA (2009). Analysis of the baseline survey on the prevalence of Salmonella in holdings with breeding pigs, in the EU, 2008. Part A: Salmonella prevalence estimates, EFSA Journal, 7(12): [93 pp.]. https://doi:10.2903.1377.

EFSA (2013). The European Union Summary Report on Trends and Sources of Zoonoses, Zoonotic Agents and Food-borne Outbreaks in 2011. EFSA Journal, 11:3129, 1-250. https://doi:10.2903/j.efsa.2013.3129.

EFSA (2014). The European Union summary report on trends and sources of zoonoses, zoonotic agents and food-borne outbreaks in 2014. EFSA Journal, 2015;13(12):4329, 1-190. https://doi:10.2903/j.efsa.2015.4329.

Fedorka-Cray, P. J., Gray, J. T., W. C. (2000). Salmonella infections in pigs. In: Wray, C., W. A. (Eds.), Salmonella in Domestic Animals, pp. 191-207 Oxon, UK.

Gil Molino, M., Risco Perez, D., Goncalves Blanco, P., Fernandez Llario, P., Quesada Molina, A., Garcia Sanchez, A., Cuesta Gerveno, J. M., Gomez Gordo, L., Martin Cano, F. E., Perez Martinez, R., Varela Fernandez, E., \& Rey Perez, J. (2019). Outbreaks of antimicrobial resistantSalmonella Choleraesuis in wild boars piglets from central-western Spain. Transboundary and Emerging Diseases, 66, 225-233. https://doi.org/10.1111/tbed.13003.

Griffith, R. W., Carlson, S. A., \& Krull A. C. (2019). Salmonellosis In: Zimmerman JJ, Karriker LA, Ramirez A, Schwartz K. J, Stevenson GW, Zhang J. Diseases of Swine 11 ${ }^{\text {th }}$ Edition (pp. 912-925). John Wiley \& Sons, Inc. https://doi:10.1002/9781119350927.

Hilbert, F., Smulders, F. J. M., Chopra-Dewasthaly, R., \& Paulsen, P. (2012). Salmonella in the wildlifehuman interface. Food Research International, 45 (2), 603-608. https://doi.org/10.1016/j.foodres.2011. 08.015.

Kureljusic, J. M., Dimitric, M. P., Vidanovic, D. S., Teodorovic, V. B., Kureljusic, B. I., Velhner, M. J., \& Karabasil, N. R. (2017). Prevalence of Salmonella enterica in slaughter pigs in Serbia: Sertyping, PFGE-grnotyping and antimicrobial resistance. Journal of Infection in Developing Countries, 11, 640-645. https://doi:10.3855/jidc.9311.

Longo, A., Losasso, C., Vitulano, F., Mastrorilli, E., Turchetto, S., Petrin, S., Mantovan, C., Dalla Pozza, M. C., Ramon, E., Conedera, G., Citterio, C. V., Ricci, A., Barco, L., \& Lettini, A. A. (2019). Insight into an outbreak of Salmonella Choleraesuis var. Kunzendorf in wild boars. Veterinary Microbiology, 238 (2019) 108423. https://doi.org/10.1016/j.vetmic.2019.108423.

Luk-in, S., Chatsuwan, T., Pulsrikarn, C., Bangtrakulnonth, A., Rirerm, U., \& Kulwichit, W. (2018). High prevalence of ceftriaxone resistance among invasive Salmonella enterica serotype Choleraesuis isolates in Thailand: the emergence and increase of CTX-M-55 in ciprofloxacin-resistant S. Choleraesuis isolates. International Journal of Medical Microbiology, https://doi.org/10.1016/j.ijmm.2018.03.008.

Methner, U., Heller, M. \& Bocklisch, H. (2010). Salmonella enterica subspecies enterica serovar Choleraesuis in a wild boar population in Germany. European Journal of Wildlife Research, 56, 493-502. https://doi.org/10.1007/s10344-009-0339-3. 
Methner, U., Merbach, S., \& Peters, M. (2018). Salmonella enterica subspecies enterica serovar Choleraesuis in a German wild boar population: occurrence and characterization. Acta Veterinaria Scandinavica, 60, 65. https://doi.org/10.1186/s13028-018-0422-4.

Pedersen, K., Sorensen, G., Lofstrom, C., Leekitcharoenphon, P., Nielsen, B., Wingstrand, A., Aarestrup, F. M., Hendriksen, R. S., \& Baggesen, D. L. (2015). Reappearance of Salmonella serovar Choleraesuis var. Kunzendorf in Danish pig herds. Veterinary Microbiology. https://doi.org/10.1016/j.vetmic.2015.01.004.

Perez, J., Astorga, R., Carrasco, L., Mendez, A., Perea, A., \& Sierra, M.A. (1999). Outbreak of salmonellosis in farmed European wild boars (Sus scrofa ferus). Veterinary Records. https://doi.org/10.1136/vr.145.16.464.

Popoff M.Y. (2001). Antigenic Formulas of the Salmonella Serovas. 8th Edn. WHO Collaborating Centre for Reference and Research onSalmonella Pasteur Institute, Paris, France, 166p.

Ruiz-Fons, F. (2017). A review of the current status of relevant zoonotic pathogens in wild swine (Sus scrofa ) populations: changes modulating the risk of transmission to humans. Transboundary and Emerging Diseases, 64, 68-88. https://doi.org/10.1111/tbed.12369.

Stojanac, N., Stevancevic, O., Potkonjak, A., Savic, B., Stancic I. \& Vracar, V. (2014). Importance of Breeding Pigs in the Spread of Salmonella on Farms. Acta Scientiae Veterinariae, 2014. 42: 1180.

Stone, G.G., Oberst, R. D., Hays, M. P., Mcvey, S., \& Chengappa M. M. (1994). Detection of Salmonella Serovars from Clinical Samples by Enrichment Broth Cultivation-PCR Procedure. Journal of Clinical Microbiology, 32(7), 1742-1749. https://doi:10.1128/JCM.32.7.1742-1749.1994.

Tizard, I. R. (2009). Immunity in the fetus and newborn. In I. R. Tizard (Ed.), Veterinary Immunology and Immunopathology, 8th ed. (pp. 223-238). Philadelphia: Elsevier.

Wills, R. W., Gray, J. T., Fedorka-Cray, P. J., Yoon, K. J., Ladely, S., \& Zimmerman, J. J. (2000). Synergism between porcine reproductive and respiratory syndrome virus (PRRSV) and Salmonella Choleraesuis in swine. Veterinary Microbiology, 71(3-4), 177-192. https://doi.org/10.1016/S0378-1135(99)00175-3.

Table 1

Susceptibility of 6 tested S. Choleraesuis isolates to antimicrobials.

\begin{tabular}{ll}
\hline Antimicrobials & Isolates $1-6$ \\
\hline Penicillin & $\mathrm{R}$ \\
Ampicillin & $\mathrm{R}$ \\
Amoxicillin & $\mathrm{R}$ \\
Cephalosporin & $\mathrm{S}$ \\
Gentamycin & $\mathrm{S}$ \\
Streptomycin & $\mathrm{R}$ \\
Neomycin & $\mathrm{S}$ \\
Colisitin & $\mathrm{S}$ \\
Enrofloxacin & $\mathrm{S}$ \\
Tetracycline & $\mathrm{R}$ \\
Florfenicol & $\mathrm{R}$ \\
Sulphonamide & $\mathrm{S}$ \\
\hline
\end{tabular}

S - Sensitive; I - Intermediate; R - Resistant

Figure 1

Pathological and histopathological findings. (a) Cyanosis of the skin of the nose, ears and ventral neck of 
a dead piglet. The skin on the tip of the ear is dry and dark purple with sloughed portions. (b) Enlarged dark purple mesenteric lymph nodes (arrows), enlarged spleen (splenomegaly) and moderately enlarged liver (hepatomegaly) were observed in most piglets necropsied. Inset: Magnification of the right hepatic lobe displaying scattered small white spots in the parenchyma (foci of parenchymal necrosis). (c) Colonic mucosa covered with multifocal adherent gray-yellow fibrinous membranes was observed in some necropsied piglets. (d) Hyperemia of liver, sinusoids infiltrated by erythrocytes, interstitial infiltration with mononuclear cell and foci of necrosis (arrow) or hemorrhage were present in liver tissue of dead piglets, haematoxylin and eosin $\mathrm{x} 220$.
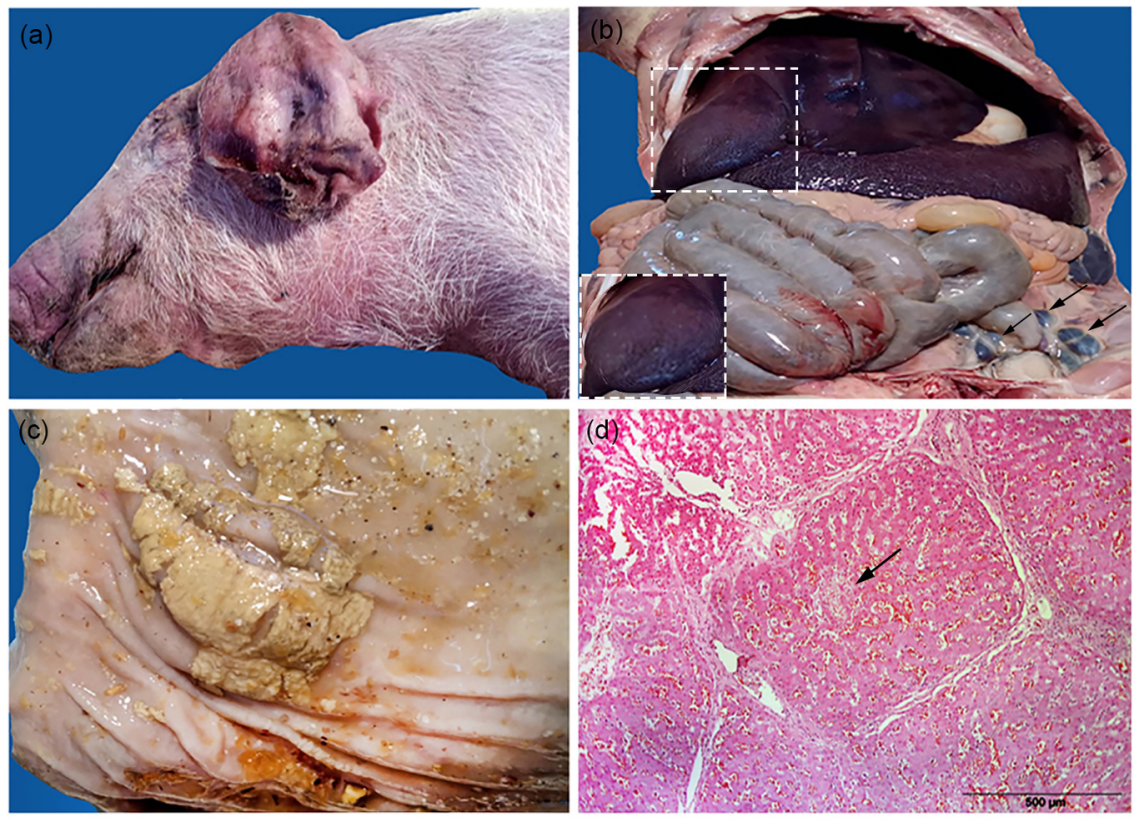